\title{
Efficacy of a nucleoside-sparing regimen of darunavir/ritonavir plus raltegravir in treatment-naive HIV-1-infected patients (ACTG A5262)
}

\author{
Babafemi Taiwo ${ }^{a}$, Lu Zheng ${ }^{b}$, Sebastien Gallien ${ }^{c}$, Roy M. Matining ${ }^{b}$, Daniel R. Kuritzkes ${ }^{c}$, \\ Cara C. Wilson $^{d}$, Baiba I. Berzins ${ }^{a}$, Edward P. Acosta ${ }^{e}$, Barbara Bastow ${ }^{f}$, Peter S. Kim ${ }^{g}$, \\ Joseph J. Eron Jrh , and ACTG A5262 Team
}

aDivision of Infectious Diseases, Northwestern University, Chicago, Illinois bStatistical Data Analysis Center, Harvard School of Public Health, Cambridge, Boston, Massachusetts 'Section of Retroviral Therapeutics, Brigham and Women's Hospital, Harvard Medical School, Cambridge, Boston, Massachusetts dDivision of Infectious Diseases, University of Colorado Health Sciences Center, Denver, Colorado eDivision of Clinical Pharmacology, Department of Pharmacology and Toxicology, University of Alabama at Birmingham, Birmingham, Alabama ${ }^{\dagger}$ ACTG Operations Center, Social \& Scientific Systems Inc., Silver Spring 9Division of AIDS, National Institute of Allergy and Infectious Diseases, National Institutes of Health, Bethesda, Maryland hDivision of Infectious Diseases, University of North Carolina at Chapel Hill, Chapel Hill, North Carolina, USA

\section{Abstract}

Objective-To explore darunavir/ritonavir (DRV/r) plus raltegravir (RAL) combination therapy in antiretroviral-naive patients.

Design-Phase IIb, single-arm, open-label, multicenter study.

Methods-One hundred and twelve antiretroviral-naive, HIV-1-infected patients received DRV/r $800 / 100 \mathrm{mg}$ once daily and RAL $400 \mathrm{mg}$ twice daily. Primary endpoint was virologic failure by week 24. Virologic failure was defined as confirmed viral load of 1000 copies $/ \mathrm{ml}$ or more at week 12 , or an increase of more than $0.5 \log _{10}$ copies $/ \mathrm{ml}$ in viral load from week 4 to 12 , or a confirmed viral load of more than 50 copies $/ \mathrm{ml}$ at or after week 24 . Protease and integrase genes were sequenced in patients experiencing virologic failure.

Results-Virologic failure rate was 16\% [95\% confidence interval (CI) 10-24] by week 24 and $26 \%$ (95\% CI 19-36) by week 48 in an intent-to-treat analysis. Viral load at virologic failure was 51-200 copies/ml in 17/28 failures. Adjusting for age and sex, virologic failure was associated

(C) 2011 Wolters Kluwer Health | Lippincott Williams \& Wilkins

Correspondence to Babafemi Taiwo, MBBS, Division of Infectious Diseases, Northwestern University, Chicago, IL 60611, USA. Tel: +1 312695 5085; fax: +1 312695 5088; b-taiwo@northwestern.edu.

Conflicts of interest

The project described was supported by Award Number U01AI068636 and U01AI68634 from the National Institute of Allergy and Infectious Diseases and supported by the National Institute of Mental Health (NIMH) and National Institute of Dental and

Craniofacial Research (NIDCR). The content is solely the responsibility of the authors and does not necessarily represent the official views of the National Institute of Allergy and Infectious Diseases or the National Institutes of Health. The project is supported in part by grants funded by the National Center for Research Resources. The authors would like to thank the study volunteers. We also thank Merck for providing raltegravir and Tibotec Therapeutics for providing darunavir.

B.T. has served as an advisor and received research support and honoraria from Tibotec. E.P.A. has served as a consultant to Tibotec and Merck. D.R.K. is a consultant to Merck and has received honoraria and research support from the company. J.J.E is a consultant to Abbott, GlaxoSmithKline, Merck, ViiV and Tibotec, and has received research support (to UNC) from GlaxoSmith-Kline and Merck. 
with baseline viral load of more than 100000 copies/ml [hazard ratio 3.76, 95\% CI (1.52-9.31), $P$ $=0.004]$ and lower CD4 cell count [0.77 per 100 cells $/ \mu 1$ increase (95\% CI $0.61-0.98), P=0.037]$. When trough RAL concentrations were included as a time-varying covariate in the analysis, virologic failure remained associated with baseline viral load more than 100000 copies/ml [hazard ratio $=4.67$ (95\% CI 1.93-11.25), $P<0.001$ ], whereas RAL level below detection limit in plasma at one or more previous visits was associated with increased hazard [hazard ratio $=3.42(95 \% \mathrm{CI}$ 1.41-8.26), $P=0.006]$. All five participants with integrase mutations during virologic failure had baseline viral load more than 100000 copies $/ \mathrm{ml}$.

Conclusion-DRV/r plus RAL was effective and well tolerated in most patients, but virologic failure and integrase resistance were common, particularly in patients with baseline viral load more than 100000 copies $/ \mathrm{ml}$.

\section{Keywords}

antiretroviral therapy; darunavir; nucleoside sparing; raltegravir

\section{Introduction}

Reverse transcriptase inhibitor (RTI) sparing antiretroviral regimens are needed given potential toxicities of some nucleos(t)ide RTIs (NRTIs) [1] and the frequency of transmitted NRTI and nonnucleoside RTI (NNRTI) resistance [2]. Two fully active antiretroviral drugs may be sufficient to suppress HIV-1 replication in treatment-naive patients [3]. Darunavir (DRV) is a potent and well tolerated protease inhibitor with no observed resistance during virologic failure when combined with two NRTIs in treatment-naive patients [4]. Raltegravir (RAL), an integrase inhibitor, is potent and well tolerated for initial therapy in combination with two NRTIs, but RAL resistance emerges in approximately one-third of patients with virologic failure [5]. AIDS Clinical Trials Group (ACTG) Study A5262 was designed to explore a two-drug, RTI-sparing regimen of DRV/ritonavir (DRV/r) plus RAL for initial antiretroviral therapy.

\section{Methods}

\section{Study participants}

A5262 participants were treatment-naive, HIV-1-infected adults ( $\geq 18$ years old) with plasma HIV-1 RNA concentration (viral load) at least 5000 copies/ml. Exclusion criteria included active hepatitis B, renal failure requiring dialysis and protocol-specified abnormal laboratory values. Patients with more than one DRV resistance-associated mutation (RAM) (V11I, V32I, L33F, I47V, I50V, I54L, I54M, T74P, I84V, and L89V), L76V alone, or known major integrase RAM (N155H, Q148H/R/K, Y143C/R, and G140S) were also excluded. Ethics review committees at each research site approved the study. Each participant provided a written informed consent.

\section{Study design and interventions}

Study participants received open-label DRV $800 \mathrm{mg}$ (two $400 \mathrm{mg}$ tablets) and ritonavir 100 $\mathrm{mg}$ (one capsule) daily plus RAL $400 \mathrm{mg}$ (one tablet) twice daily. The site investigator was permitted to construct a new regimen if a patient experienced virologic failure or treatmentlimiting adverse effects.

\section{Procedures and assessments}

Study participants had screening, preentry, and entry visits (day 0). Subsequent evaluations occurred at weeks $1,4,12,24,36,48$, and 52 . At entry and weeks $4,12,24,36$, and 48, viral load, hematology, liver function tests, and blood chemistries were analyzed. Viral load was 
also determined at week 1 in all participants and at week 52 in participants with suspected virologic failure at week $48 . \mathrm{CD}^{+}$and $\mathrm{CD}^{+} \mathrm{T}$-cell counts were determined at entry and weeks 12, 24, 36, and 48. Fasting lipid levels were measured at entry and weeks 24 and 48. Adherence was assessed at weeks 1, 4, 12, 24, and 48 by self-report (number of missed doses over a 4-day recall) [6]. Plasma samples for trough concentrations $\left(C_{\text {trough }}\right)$ of RAL and DRV were stored at each visit that had an adherence assessment. Participants with suspected virologic failure were asked to return for a failure confirmation visit within 7-35 days of collecting the initial failure sample. At the failure confirmation visit, adherence was assessed and samples collected for viral load, protease and integrase genotype, T-cell counts, and $C_{\text {trough }}$.

Viral load was measured centrally using the Abbott RealTime HIV-1 Test on the m2000 system (Abbott RT/m2000 assay) (Abbott Molecular Inc., Des Plaines, Illinois, USA). Population sequencing of the integrase and protease genes was performed during virologic failure. Genotypic mutations according to the 2009 International AIDS Society (IAS) USA [7] plus G140S for integrase were considered. DRV and RAL plasma concentrations were determined using internally and externally validated mass spectrometry (RAL) and ultraperformance liquid chromatography (DRV) methods. All interday and intraday variability was less than 10\%; the lower limits of detection for RAL and DRV were 10 and $50 \mathrm{ng} / \mathrm{ml}$, respectively.

An independent Study Monitoring Committee reviewed study conduct, safety and efficacy approximately 24 weeks after enrollment of the 40th participant. To assess the impact of assay variation on viral load determinations, stored plasma samples from the first 10 patients with low-level viremia (viral load 51-200 copies/ml) during virologic failure were retested posthoc using the Roche Amplicor HIV-1 Monitor Test, version 1.5 (Roche Diagnostic Systems, Branchburg, New Jersey, USA).

\section{Outcome measures}

The primary endpoint was virologic failure prior to or at week 24 . Virologic failure was defined as confirmed viral load 1000 copies/ml or more at week 12, or an increase of more than $0.5 \log _{10}$ copies $/ \mathrm{ml}$ in viral load from week 4 to 12 (including rebound to $>50$ copies/ $\mathrm{ml}$ from week 4 to 12 for patients with week 4 value 50 copies $/ \mathrm{ml}$ ), or confirmed viral load more than 50 copies $/ \mathrm{ml}$ at or after week 24 . Secondary outcome measures included virologic failure through week 48, viral load less than 50 or less than 200 copies/ml at weeks 24 and 48 , incidence of adverse events that was at least grade 3 (severe) or any grade if it led to permanent drug discontinuation, changes in fasting lipid concentrations, protease or integrase inhibitor resistance during virologic failure, adherence to study treatment, $C_{\text {trough }}$ of RAL and DRV, and changes in CD4 ${ }^{+} \mathrm{T}$-cell counts (CD4 cell count).

\section{Statistical analysis}

For the primary analysis, the cumulative proportion of participants experiencing virologic failure at or before week 24 and corresponding two-sided 95\% confidence interval (CI) were estimated using the Kaplan-Meier method and Greenwood's formula [8]. The primary efficacy analysis used the intent-to-treat (ITT) approach, including all treated patients regardless of study treatment modification and censoring follow-up if a participant was lost to follow-up or died without previously meeting the definition of virologic failure. Enrolled participants who never started the study treatment were excluded. Log-rank test and Cox proportional hazards regression were used to assess predictors of virologic failure.

Acceptable virologic failure rate was estimated using week 24 virologic failure rates observed in large phase III trials of current preferred regimens: efavirenz plus two-NRTI 
arm of ACTG A5142 (22\%) [3]; efavirenz plus two-NRTI arm of ACTG A5095 (17\%) [9]; and tenofovir/emtricitabine (TDF/FTC) plus RAL (10-15\%) [10]. It was prespecified that RAL plus DRV/r would be considered satisfactory if the upper bound of the CI for cumulative virologic failure at week 24 was less than $35 \%$. With a sample size of 100 evaluable participants, the study was estimated to have $91 \%$ probability that the upper bound of the two-sided $95 \% \mathrm{CI}$ for the virologic failure rate would be less than $35 \%$ if the underlying true failure rate was $20 \%$. The planned sample size was increased by $10 \%$ to 111 participants to account for loss to follow-up.

Proportions of participants with viral load less than 200 and less than 50 copies $/ \mathrm{ml}$ at weeks 24 and 48 were estimated using ITT (missing/off study without virologic failure ignored) and modified ITT (missing/off treatment considered failure) approaches.

Adherence was classified as perfect (zero reported missed doses) or imperfect (any missed doses of DRV/r or RAL, failure to answer the 4-day recall question, or missed the study visit). Pharmacokinetic analyses included geometric mean of trough concentrations $\left[C_{\text {trough(avg) }}\right]$ obtained within a defined window (within 9-15 and 20-28 h after the last dose for RAL and DRV, respectively). Only $C_{\text {trough }}$ up to and including the time of virologic failure confirmation visit was included for participants with virologic failure. $C_{\text {trough }}$ below the assay detection limits was replaced with half of the corresponding lower limit of quantification. Sensitivity analysis examined $C_{\text {trough(avg) }}$ using all available $C_{\text {trough }}$ values. Adverse events were graded according to the severity scale of the Division of AIDS, National Institutes of Health [11]. Safety analyses used as-treated approach, including all patients who initiated study treatment and censoring follow-up at treatment discontinuation.

Baseline viral load level and CD4 cell count were calculated as the geometric and arithmetic means, respectively, of preentry and entry evaluations. Changes in continuous measures from baseline were assessed by Wilcoxon signed rank test. Comparisons between participants with and without virologic failure used Wilcoxon rank sum test for continuous measures, Fisher's exact test for binary measures and Cochran-Armitage test for ordered categorical measures.

All $P$ values and CIs presented were two-sided and nominal, unadjusted for interim analysis and multiple comparisons. Analyses were done using SAS, version 9.2 (SAS Institute, Cary, North Carolina, USA), StatXact 8 PROCs (Cytel, Cambridge, Massachusetts, USA), and Splus, version 6 (Insightful, Seattle, Washington, USA).

\section{Results}

\section{Study participants}

A total of 113 patients were enrolled at 22 sites in the USA. One participant did not initiate study drugs and was taken off study. Of 112 participants who initiated DRV/r plus RAL with a median age of 36 years, $88 \%$ were men and $44 \%$ were white non-Hispanic. Median CD4 cell count and viral load were 271 cells/ $\mu 1$ and $4.87 \log _{10}$ copies $/ \mathrm{ml}$, respectively (Table 1). Forty-nine (44\%) participants had baseline viral load more than 100000 copies/ $\mathrm{ml}$ including six (5\%) with levels higher than 750000 copies $/ \mathrm{ml}$. Pretreatment antiretroviral drug resistance was detected in 21 (19\%) participants: nine with (8\%) NNRTI, eight (7\%) with NRTI, two (2\%) with protease inhibitor, one $(1 \%)$ with NRTI plus NNRTI, and one (1\%) with NRTI plus NNRTI and protease inhibitor mutations. No participant had a DRV RAM. Ninety-seven (87\%) participants completed 52 weeks follow-up. Fifteen (13\%) participants discontinued participation due to inability to get to clinic (seven), inability of study staff to reach participant (four), withdrawal of consent (two), unwillingness to adhere to study requirements (one), and death (one). 


\section{Efficacy}

Seventeen participants $(16 \%, 95 \%$ CI 10-24) experienced virologic failure by week $24: 11$ failed to suppress viral load (one with $>1000$ copies $/ \mathrm{ml}$ at week 12; 10 with $>50$ copies $/ \mathrm{ml}$ at week 24) and six due to viral rebound. Eleven participants experienced virologic failure (due to viral load rebound to $>50$ copies/ml) after week 24 . Thus, virologic failure occurred in 28 participants by week 48 (Table 2); virologic failure rate by week 48 was $26 \%$ (95\% CI 19-36). Three participants with virologic failure subsequently attained viral load less than 50 copies/ml without changing therapy. In ITT analysis, viral load was less than 50 copies/ $\mathrm{ml}$ in 79\% (95\% CI 70-86) of participants at week 24 and in 71\% (95\% CI 61-79) at week 48; using modified ITT analysis, viral load was less than 50 copies $/ \mathrm{ml}$ in $74 \%$ (95\% CI 6682) of participants at week 24 and in 61\% (95\% CI 52-70) at week 48 (Fig. 1). Viral load less than 200 copies/ml was achieved in $93 \%$ (95\% CI 87-97) at week 24 and $86 \%$ (95\% CI 78-92) at week 48 in ITT analysis and in 88\% (95\% CI 81-94) and 73\% (95\% CI 65-81) at week 24 and 48 in the modified ITT analysis.

Participants with virologic failure had higher baseline viral load (median 5.22 vs. $4.70 \log _{10}$ copies $/ \mathrm{ml}, P=0.002$ ) and lower baseline CD4 cell count (192 vs. 322 cells $/ \mu 1, P=0.007$ ) compared with those who did not experience virologic failure (Table 1). Of the 28 participants with virologic failure, 21 had baseline viral load more than 100000 copies $/ \mathrm{ml}$ and these patients had more rapid time to virologic failure $(P<0.001)$ (Fig. 2$)$. Multivariable model for time to virologic failure, adjusting for age and sex, demonstrated that virologic failure was associated with baseline viral load more than 100000 copies $/ \mathrm{ml}$ (hazard ratio $=3.76,95 \%$ CI $1.52-9.31, P=0.004$ ) and lower CD4 cell count (hazard ratio $=0.77,95 \%$ CI $0.61-0.98$ per 100 cells $/ \mu 1$ increase, $P=0.037)$. Seventeen $(15 \%)$ participants were classified as having imperfect adherence. No association was detected between virologic failure and adherence, age, sex, intravenous drug use, race/ethnicity, or presence of any mutation at baseline (all $P>0.10$ ).

Seventeen participants with virologic failure (61\%) had viral load of 51-200 copies/ml at the first viral load determination during virologic failure, four (14\%) had 201-1000 copies/ml and seven $(25 \%)$ had more than 1000 copies/ml. Viral load levels in the first 10 patients with low-level viremia during virologic failure were similar when determined using the Roche HIV Monitor v1.5 and Abbott RT/m2000 assays. Baseline CD4 cell count, viral load, resistance, adherence, and detection of mutations at the time of virologic failure were not significantly different between participants who failed with viral load higher than 200 vs. 51-200 copies/ml (all $P \geq 0.40$ ). Participants with virologic failure as a result of failure to suppress viremia vs. viral rebounders were similar with respect to baseline characteristics (age, sex, race/ethnicity, intravenous drug use, CD4 cell count, and viral load) and RAL and DRV trough concentrations/detectability, adherence (all $P>0.10$ ).

\section{HIV-1 drug resistance}

Integrase resistance testing was successful in 25 of the 28 virologic failures. All five participants with evidence of integrase RAMs [N155H (one), N155H/N (two), Q148Q/R, and N155H/N (one), Q148K/Q and N155H/N (one)] had baseline viral load more than 100 000 copies $/ \mathrm{ml}$. None of these patients had documented treatment interruption. No new protease inhibitor RAMs were detected in the 23 participants with successful protease sequencing following virologic failure.

\section{Pharmacokinetics}

Considering the defined trough period, median (quartile 1-quartile 3) $C_{\text {trough(avg) was }} 1218$ (789-1809) $\mathrm{ng} / \mathrm{ml}$ for DRV and 117 (52-250) ng/ml for RAL (Supplementary Table 1, http://links.lww.com/QAD/A177). DRV and RAL $C_{\text {trough(avg) }}$ values within the defined 
trough period were not significantly different for patients with and without virologic failure, perfect vs. imperfect adherence, viral load more than $200 \mathrm{vs.} \mathrm{51-200} \mathrm{copies} / \mathrm{ml}$ at the time of virologic failure, and presence or absence of resistance mutations at virologic failure (all $P>0.10$ ). Similar results were obtained in sensitivity analyses that included all available DRV and RAL $C_{\text {trough }}$ except for DRV $C_{\text {trough(avg), which was lower in those with virologic }}$ failure (1042 vs. $1649 \mathrm{ng} / \mathrm{ml}, P=0.017$ ). For RAL, at least one $C_{\text {trough }}$ below detection limits (BDLs) occurred in 10/27 (37\%) participants with virologic failure compared with seven of $76(9 \%)$ nonvirologic failure participants $(P=0.002)$; for DRV, five of $21(24 \%)$ virologic failure participants had BDL levels compared to two of 62 (3\%) nonvirologic failure participants $(P=0.01)$ (Table 3$)$. When adjusted for DRV or RAL $C_{\text {trough }}$ [either continuous or categorical variable (BDL or not)] as a time-varying covariate with one-study-visit lag (at the visit immediately before or at all previous visits) in Cox PH models, baseline viral load more than $100000 \mathrm{copies} / \mathrm{ml}$ remained significantly associated with hazard of virologic failure $(P<0.05)$. More specifically, when RAL $C_{\text {trough }}$ was evaluated as a categorical variable, having RAL $C_{\text {trough }}$ BDL within the defined trough period at the visit immediately before was associated with increased hazard of virologic failure (hazard ratio $=5.25,95 \% \mathrm{CI}$ $1.41-19.58, P=0.014]$ and the hazard ratio was 5.05 (95\% CI 1.64-15.56) $(P=0.005)$ for baseline viral load; the hazard ratio for having RAL $C_{\text {trough }} \mathrm{BDL}$ at one or more previous visits was $3.42(95 \%$ CI $1.41-8.26)(P=0.006)$ and 4.67 (95\% CI $1.93-11.25)(P<0.001)$ for baseline viral load. Marginally significant association was found between virologic failure and a DRV $C_{\text {trough }}$ BDL within the defined trough window at the visit immediately before (hazard ratio $=4.28,95 \%$ CI 0.92-20.04, $P=0.065$; baseline viral load hazard ratio $=3.75$, 95\% CI 1.13-12.41, $P=0.030)$. The association became significant when all DRV $C_{\text {trough }}$ were included (hazard ratio $=3.89,95 \%$ CI $1.32-11.49, P=0.014$; baseline viral load hazard ratio $=4.34,95 \%$ CI $1.73-10.86, P=0.002)$.

\section{Immunological outcomes}

The median CD4 cell count increase from baseline was 142 (80-196) cells/ $\mu 1$ at week 24 and $200(114-318)$ cells $/ \mu$ l at week 48 (all $P<0.001$ ) and were similar at week 24 in patients with baseline viral load less than or equal to or more than 100000 copies $/ \mathrm{ml}(P>0.1)$. At week 48 , the median increase was 233 cells $/ \mu$ in patients with baseline viral load higher than 100000 copies $/ \mathrm{ml}$ vs. 180 cells $/ \mu$ l in those with viral load 100000 copies $/ \mathrm{ml}$ or less $(P$ $=0.044)$.

\section{Safety and tolerability}

Twenty-one participants (19\%) reported at least one grade 3 (severe) or higher clinical or laboratory adverse events, five of which were classified as possibly related to study treatment: dyslipidemia (three), diabetes mellitus (one), and elevated aspartate transaminase/ alanine transaminase (one). No events were considered probably or definitely related to study treatment. One participant permanently discontinued study treatment due to grade 2 (moderate) maculopapular rash and abdominal pain. Death occurred in one patient at week 9 from cryptosporidiosis.

Median increases in fasting high-density lipoprotein (HDL), low-density lipoprotein, total cholesterol, and triglycerides from baseline to week 48 were $9,17,30$, and $23 \mathrm{mg} / \mathrm{dl}$, respectively $(P<0.001$, except triglycerides $P=0.006)$. Fasting total cholesterol: HDL ratio did not change significantly from baseline ( 0.40 at weeks 24 and 48$)$.

\section{Discussion}

In HIV-1-infected treatment-naive participants enrolled in A5262, an RTI-free, two-drug regimen comprising DRV/r plus RAL met the protocol definition of acceptable virologic 
efficacy (at week 24), but only 71 and $61 \%$ of participants had viral load less than 50 copies/ $\mathrm{ml}(86$ and $73 \%<200$ copies $/ \mathrm{ml})$ at week 48 in ITT and modified ITT analyses, respectively. Baseline viral load more than 100000 copies $/ \mathrm{ml}$ was associated with an increased risk of virologic failure. Baseline CD4 cell count per 100-cell increase was associated with reduced risk of virologic failure. In multivariable models fitted with DRV or RAL $C_{\text {trough }}$ BDL, baseline viral load more than 100000 copies/ml remained strongly associated with increased risk of virologic failure. Having RAL $C_{\text {trough }} \mathrm{BDL}$ at the visit immediately before or at one or more previous visits was also associated with an increased hazard of failure.

Potential explanations for our findings were explored. Self-reported adherence (4-day recall) was not significantly different between those with and without virologic failure, or between those with baseline viral load less than or equal to or more than 100000 copies $/ \mathrm{ml}$. However, having one or more DRV and RAL plasma concentrations below detection limits was significantly more common in those with virologic failure, possibly related to unreported suboptimal adherence. Other investigators have demonstrated discordance between self-reported adherence and objectively measured adherence [12]. Adverse effects of therapy are unlikely to have been the major determinant of adherence or virologic efficacy as RAL and DRV were well tolerated. An alternative hypothesis is that asymmetrical dosing of DRV/r (once daily) and RAL (twice daily) predisposed to suboptimal adherence and virologic failure, but such association has not emerged as a concern with RAL twice daily plus TDF/FTC, a similarly asymmetrically dosed regimen [13]. It was suggested recently that RAL-DRV interactions may lower plasma concentrations of DRV [14], but DRV $C_{\text {trough }}$ observed in this study (Supplementary Table 1, http://links.lww.com/QAD/A177) are within the range reported in an intensive pharmacokinetic study of DRV 800/100 mg daily [15]. Finally, because over half of the patients who experienced virologic failure had low-level viremia (51-200 copies $/ \mathrm{ml})$ at the time of failure, we considered virologic failure artifacts due to assay variability [16]. This possibility was excluded because viral load determinations during low-level viremia were similar with the Abbott m2000 and the Roche Amplicor Ultrasensitive Assays in the first 10 participants with low-level virologic failure.

An association between efficacy and baseline viral load has been demonstrated with other antiretroviral regimens. In some but not all studies of two NRTIs plus a third preferred agent, smaller proportions of patients with baseline viral load more than $100000 \mathrm{copies} / \mathrm{ml}$ achieved HIV RNA less than 50 copies/ml at 48 weeks [5,13,17-21], but these differences tend to be small, are in part related to tolerability and associated with low CD4 cell count [16], and may not be synonymous with subsequent virologic failure [22]. In our RTI-sparing study in which we specifically examined virologic failure, as opposed to a combined endpoint, the differences in virologic outcomes between the high and low viral load strata were striking and the results were consistent or even more evident in multivariable analyses that included baseline CD4 cell count or assessments of drug concentrations. A pilot study evaluating twice-daily atazanavir (ATV) plus RAL was prematurely terminated at week 24 due in part to adverse events and frequent RAL resistance in those with virologic failure [23]. A larger randomized study, however, found no significant difference in viral load less than 40 copies/ml at week 48 in patients treated with RAL plus lopinavir/ritonavir (LPV/r) compared with TDF/FTC plus LPV/r [24]. The mean baseline viral load in the latter study was $4.25 \log _{10}$ copies $/ \mathrm{ml}$, which is lower than the $4.9 \log _{10}$ copies $/ \mathrm{ml}$ in the twice-daily RAL plus ATV study and $4.83 \log _{10}$ copies/ml in our study. Our study is also the first to report virologic outcomes by baseline viral load less than or equal to or more than 100000 copies/ml separately from nonvirologic treatment discontinuations, further limiting crossstudy comparisons. The mechanisms underlying the poorer virologic outcomes in some patients with high baseline viral load, as observed in this study, are uncertain. One possibility is that high baseline viral load may be associated with more extensive reservoir 
of infected cells and prolonged viral decay time to levels below 50 copies $/ \mathrm{ml}$. However, only one of the 28 virologic failures in the current study had viral dynamics that may be explained solely by this specific hypothesis. Another possibility is that high baseline viral load may predispose to greater diversity of HIV-1 quasispecies and an increased opportunity to select drug-resistant mutants. Q148R minority variants were detected at very low levels (median $0.46 \%$ ) in $86 \%$ of treatment-naive patients in one study [25]. The effect of pretherapy RAL-resistant minority variants on virologic outcome in treatment-experienced patients has not been clearly demonstrated $[25,26]$ and to our knowledge has not yet been reported in treatment-naive patients.

Baseline viral load more than 100000 copies $/ \mathrm{ml}$ appears to increase the risk of RAL resistance in patients receiving DRV/r plus RAL. All the patients with evidence of RAL resistance mutations at virologic failure (20\% of those genotyped) had baseline viral load more than 100000 copies $/ \mathrm{ml}$. None of these patients had documented treatment interruption, and no significant difference in RAL $C_{\text {trough }}$ was observed between those with or without RAL resistance. Viral load at the time of integrase genotyping in the five participants ranged from 62 to 685 copies/ml. Notably, a participant who achieved HIV RNA less than 50 copies $/ \mathrm{ml}$ at week 12 and had no subsequent documentation of viral load level more than 100 copies/ml experienced virologic failure at week 48 with detection of Q148K/Q and N155H/N. Thus, like NNRTI and NRTI resistance mutations [27], RAL resistance mutations may be present during low-level viremia [28], an important observation as recent guidelines state that viral load more than 200 copies $/ \mathrm{ml}$ can be considered the threshold for virologic failure in clinical practice [1]. Protease inhibitor resistance was not detected in any participant experiencing virologic failure, consistent with evidence that boosted protease inhibitor resistance seldom develops early in virologic failure [29].

Interpretation of this study should take into account its single-arm design as a randomized trial could have reduced the potential impact of patient characteristics and other variables. Also, patients were not screened for pretreatment RAL resistance, but primary mutations that confer resistance to RAL are uncommon in RAL-naive patients [30]. Despite these limitations, the results of A5262 raise important issues that should be examined carefully in future clinical trials evaluating DRV/r plus RAL and perhaps in all RTI-sparing two-drug regimen trials. We urge caution in patients with baseline viral load more than 100000 copies $/ \mathrm{ml}$ and emphasize a need to further elucidate the implications of low-level viremia in patients receiving the regimen.

\section{Supplementary Material}

Refer to Web version on PubMed Central for supplementary material.

\section{Acknowledgments}

B.T. and J.J.E. conceived the study. D.R.K was the protocol virologist and EPA the protocol pharmacologist. Substantial contributions to study design and interpretation of the data were made by B.T., L.Z., R.M.M., D.R.K., C.C.W., E.P.A., P.S.K., and J.J.E. L.Z., and R.M.M. analyzed the data. S.G. performed viral resistance assays. B.I.B. was the Field Representative and B.B. was the Clinical Trials Specialist. All authors had full access to the data and vouch for the accuracy and completeness of the data and analyses. Initial drafts of the manuscript written by B.T., L.Z., R.M.M. and J.J.E. were reviewed, edited and approved by all of the authors.

\section{References}

1. Panel on Antireroviral Guidelines for Adults and Adolescents. Guidelines for the use of antiretroviral agents in HIV-1 infected adults and adolescents. Department of Health and Human Services; 2011. http://aidsinfo.nih.gov/contentfiles/AdultandAdolescentGL.pdf 
2. Wittkop L, Gunthard HF, de Wolf F, Dunn D, Cozzi-Lepri A, de Luca A, et al. Effect of transmitted resistance on virological and immunological response to initial combination antiretroviral therapy for HIV (EuroCoord-CHAIN joint project): a European multicohort study. Lancet Infect Dis. 2011; 11:363-371. [PubMed: 21354861]

3. Riddler SA, Haubrich R, DiRienzo AG, Peeples L, Powderly WG, Klingman KL, et al. Classsparing regimens for initial treatment of HIV-1 infection. N Engl J Med. 2008; 358:2095-2106. [PubMed: 18480202]

4. Mills AM, Nelson M, Jayaweera D, Ruxrungtham K, Cassetti I, Girard PM, et al. Once-daily darunavir/ritonavir vs. lopinavir/ritonavir in treatment-naive, HIV-1-infected patients: 96-week analysis. AIDS. 2009; 23:1679-1688. [PubMed: 19487905]

5. Lennox JL, DeJesus E, Berger DS, Lazzarin A, Pollard RB, Ramalho Madruga JV, et al. Raltegravir versus efavirenz regimens in treatment-naïve HIV-1 infected patients: 96 week efficacy, durability, subgroup, safety and metabolic analyses. J Acquir Immune Defic Syndr. 2010; 55:39-48. [PubMed: 20404738]

6. Chesney MA, Ickovics JR, Chambers DB, Gifford AL, Neidig J, Zwickl B, Wu AW. Self-reported adherence to antiretroviral medications among participants in HIV clinical trials: the AACTG adherence instrument. Patient Care Committee and Adherence Working Group of the Outcomes Committee of the Adult AIDS Clinical Trials Group (AACTG). AIDS Care. 2000; 12:255-266. [PubMed: 10928201]

7. Johnson VA, Brun-Vezinet F, Clotet B, Gunthard HF, Kuritzkes DR, Pillay D, et al. Update of the drug resistance mutations in HIV-1: December 2009. Top HIV Med. 2009; 17:138-145. [PubMed: 20068260]

8. Kalbfleisch, JD.; Prentice, RL. The statistical analysis of failure time data. New York: Wiley; 1980.

9. Markowitz M, Nguyen BY, Gotuzzo E, Mendo F, Ratanasuwan W, Kovacs C, et al. Rapid and durable antiretroviral effects of the HIV-1 integrase inhibitor raltegravir as part of combination therapy in treatment-naïve patients with hIV-1 infection: results of a 48-week controlled study. J Acquir Immune Defic Syndr. 2007; 46:125-133. [PubMed: 17721395]

10. Gulick RM, Ribaudo HJ, Shikuma CM, Lalama C, Schackman BR, Meyer WA 3rd, et al. Threevs four-drug antiretroviral regimens for the initial treatment of HIV-1 infection: a randomized controlled trial. JAMA. 2006; 296:769-781. [PubMed: 16905783]

11. [Accessed 2 April 2011] Division of AIDS Table for Grading the Severity of Adult and Pediatric Adverse Events. Dec. 2004 http://www.niaid.nih.gov/LabsAndResources/resources/ DAIDSClinRsrch/Documents/daidsaegradingtable.pdf

12. Brouwer ES, Napravnik S, Smiley SG, Corbett AH, Eron JJ. Self-report of current and prior antiretroviral drug use in comparison to the medical record among HIV-infected patients receiving primary care. Pharmacoepidemiol Drug Safety. 2011; 20:432-439.

13. Eron, J.; Rockstroh, J.; Reynes, J.; Andrade-Villanueva, J.; Madruga, J.; Zhao, J., et al. QDMRK, a phase III study of the safety and efficacy of once daily (QD) versus twice daily (BID) raltegravir (RAL) in combination therapy for treatment-naïve HIV-infected patients. Abstracts of 18th Conference on Retroviruses and Opportunistic Infections; Boston, MA. February 27 to March 2 2011; p. abstract 150L

14. Fabbiani M, Di Giambenedetto S, Ragazzoni E, D’Ettorre G, Parruti G, Prosperi M, et al. Darunavir/ritonavir and raltegravir coadministered in routine clinical practice: Potential role for an unexpected drug interaction. Pharmacol Res. 2011; 63:249-253. [PubMed: 21130167]

15. Boffito, M.; Moyle, M.; Hill, A.; Sekar, V.; Lefebvre, E.; De Pauw, M., et al. The pharmacokinetic profile of darunavir with low-dose ritonavir (DRV/r) in various multiple-dose regimens over 120 h. In: Abstracts of the. 9th International Workshop on Clinical Pharmacology of HIV Therapy; New Orleans. 7-9 April 2008; p. abstract P31

16. Yan CS, Hanafi I, Kelleher AD, Carr AD, Amin J, McNally LP, et al. Lack of correlation between three commercial platforms for the evaluation of human immunodeficiency virus type 1 (HIV-1) viral load at the clinically critical lower limit of quantification. J Clin Virol. 2010; 49:249-253. [PubMed: 20884287]

17. Molina JM, Andrade-Villanueva J, Echevarria J, Chetchotisakd P, Corral J, David N, et al. Oncedaily atazanavir/ritonavir versus twice-daily lopinavir/ritonavir, each in combination with tenofovir and emtricitabine, for management of antiretroviral-naïve HIV-1-infected patients: 48 
weeks efficacy and safety results of the CASTLE study. Lancet. 2008; 372:646-655. [PubMed: 18722869]

18. Ortiz R, DeJesus E, Khanlou H, Voronin E, van Lunzen J, Andrade-Villanueva J, et al. Efficacy and safety of once-daily darunavir/ritonavir versus lopinavir/ritonavir in treatment-naive HIV-1infected patients at week 48. AIDS. 2008; 22:1389-1397. [PubMed: 18614861]

19. Sax P, Tierney C, Collier AC, Fischl MA, Mollan K, Peeples L, et al. Abacavir-lamivudine versus tenofovir-emtricitabine for initial HIV-1 therapy. N Engl J Med. 2009; 361:2230-2240. [PubMed: 19952143]

20. Smith KY, Patel P, Fine D, Bellos N, Sloan L, Lackey P, et al. Randomized, double-blind, placebo-matched, multicenter trial of abacavir/lamivudine or tenofovir/emtricitabine with lopinavir/ritonavir for initial HIV treatment. AIDS. 2009; 23:1547-1556. [PubMed: 19542866]

21. van Leth F, Andrew S, Grinsztejn B, Wilkins E, Lazanas MK, Lange JM, Montaner J. 2NN study group. The effects of baseline CD4 cell count and HIV-1 viral load on the efficacy and safety of nevirpaine or efavirenz-based first-line HAART. AIDS. 2005; 19:463-471. [PubMed: 15764851]

22. Ribaudo, H.; Lennox, J.; Currier, J.; Kuritzkes, D.; Gulick, R.; Haubrich, R., et al. Virologic failure endpoint definition in clinical trials: is using HIV-1 RNA threshold $<200$ copies/mL better than < 50 copies/mL? An analysis of ACTG studies. Abstracts of 16th Conference on Retroviruses and Opportunistic Infections; Montreal. 8-11 February 2009; p. abstract 580

23. Kozal, MJ.; Lupo, S.; DeJesus, E.; Molina, J.; McDonald, C.; Raffi, F., et al. The SPARTAN Study: A pilot study to assess the safety and efficacy of an investigational NRTI-and RTV-sparing regimen of atazanavir (ATV) experimental dose of $300 \mathrm{mg}$ bid plus raltegravir $400 \mathrm{mg}$ bid ((ATV + RAL_ in treatment-naïve HIV-1 infected subjects. Abstracts of XVIII International AIDS Conference; Vienna. 18-23 July 2010; p. abstract MOAB010

24. Reynes, J.; Lawal, A.; Pulido, F.; Soto-Malave, R.; Gathe, J.; Tian, M., et al. Lopinavir/ritonavir combined with raltegravir demonstrated similar antiviral efficacy and safety as lopinavir/ritonavir combined with tenofovir disoproxil fumarate/emtricitabine in treatment naive HIV-1 infected subjects: PROGRESS 48 week results. Abstracts of XVIII International AIDS Conference; Vienna. 18-23 July 2010; p. abstract THLBB204

25. Charpentier C, Laureillard D, Piketty C, Tisserand P, Batisse D, Karmochkine M, et al. High frequency of integrase Q148R minority variants in HIV-infected patients naïve of integrase inhibitors. AIDS. 2010; 24:867-873. [PubMed: 20160635]

26. Lui J, Miller MD, Danovich RM, Vandergrift N, Cai F, Hicks CB, et al. Analysis of low-frequency mutations associated with drug resistance to raltegravir before antiretroviral treatment. Antimicrob Agents Chemother. 2011; 55:1114-1119. [PubMed: 21173185]

27. Taiwo B, Gallien S, Aga E, Ribaudo H, Haubrich R, Kuritzkes DR, Eron JJ. Antiretroviral drug resistance in HIV-1-infected patients experiencing persistent low-level viremia during first-line therapy. J Infect Dis. 2011; 204:515-520. [PubMed: 21791652]

28. Gallien S, Delaugerre C, Charreau I, Braun J, Boulet T, Barrail-Tran A, et al. Emerging integrase inhibitor resistance mutations in raltegravir-treated HIV-1-infected patients with low-level viremia. AIDS. 2011; 25:655-659.

29. Hirsch MS, Gunthard HF, Schapiro JM, Brun-Vezinet F, Clotet B, Hammer SM, et al. Antiretroviral drug resistance testing in adult HIV-1 infection: 2008 recommendations of an International AIDS Society-USA panel. Clin Infect Dis. 2008; 47:266-285. [PubMed: 18549313]

30. Young B, Fransen S, Greenberg KS, Thomas A, Martens S, St Clair M, et al. Transmission of integrase strand-transfer inhibitor multidrug resistant HIV: case report and natural history of response raltegravir-containing antiretroviral therapy. Antiviral Ther. 2011; 16:253-256.

\section{Appendix}

A5262 study team members: Sarah W. Read, Jennifer Janik, Debra S. Meres, Michael M. Lederman, Lori Mong-Kryspin, Karl E. Shaw, Louis G. Zimmerman, Randi Leavitt (Merck), Guy De La Rosa (Tibotec), Amy Jennings.

ACTG site investigators: Karen Coleman and Meredith Rathert [Northwestern University (Site 2701) CTU Grant AI069471]; Edward Seefried and Leticia Muttera [University of 
California San Diego (Site 701) CTU Grant number AI 69432]; Michael F. Para and Heather Harber [The Ohio State University (Site 2301) CTU Grant AI069474]; Robert Kalayjian and Ann Marie Anderson [MetroHealth Medical Center (Site 2503) CTU Grant AI-069501]; Kerry Upton and Jenna White [Alabama Therapeutics CRS (Site 5801) CTU Grant U01 AI069452]; Pablo Tebas and Aleshia Thomas [University of Pennsylvania (Site 6201) CTU Grant U01-AI-69467-05, CFAR Grant P30-AI-045008-12]; Annie Luetkemeyer and Jay Dwyer [UCSF AIDS CRS (Site 801) CTU Grant 5UO1 AI069502]; Mariea Snell and James Conner [Washington University in St. Louis (Site 2101) CTU Grant AI 069495]; Nathan M. Thielman and Jacquelin Granholm [Duke University Medical Center CRS (Site 1601) CTU Grant 5U01 AI069484]; Carl J Fichtenbaum and Eva Moore [University of Cincinnati (Site 2401) CTU Grant 1U01AI069513]; David Currin and Megan Avots [UNC AIDS Clinical Trials Unit UNC AIDS CRS (Site 3201) CTU Grant 5-U01 AI069423, CTSA Grant UL 1RR 025747, CFAR Grant AI50410]; Roberto C. Arduino and Maria Laura Martinez [Houston AIDS Research (HART) (Site 31473) CTU Grant 1U01AI069503]; Mary Albrecht and Amanda Youmans [Beth Israel Deaconess (Partners/Harvard) CRS (Site 103) CTU Grant U01 AI069472-05]; Debbie Slamowitz and Sandra Valle [Stanford University AIDS CTU (Site 501) CTU Grant AI069556]; Princy N. Kumar and Joseph Timpone [Georgetown University (Site 1008) Grant 5U01AI069494]; Christine Hurley and Roberto Corales [AIDS Care (Site 1108) CTU Grant U01AI069511-02 (as of 2/12/08), CTSI Grant UL1 RR 024160]; Vicki Bailey and Husamettin Erdem [Vanderbilt Therapeutics CRS (Site 3652) CTU Grant AI-069439, Grant RR-024975]; Sharon Riddler and Sally McNulty [Pittsburgh CRS (Site 1001) CTU Grant 1 UO1 AI 069494-01]; Barbara Philpotts and Dawn Antosh [Case CRS (Site 2501) CTU Grant AI69501].

A5262 is registered with ClinicalTrials.gov (NCT00830804). 

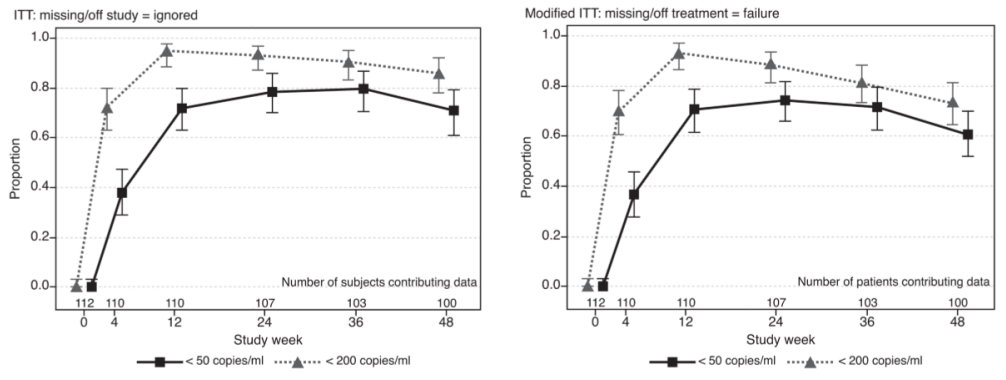

Fig. 1.

Proportion of participants with HIV-1 RNA level less than 50 and less than 200 copies/ml. 
(a) Overall

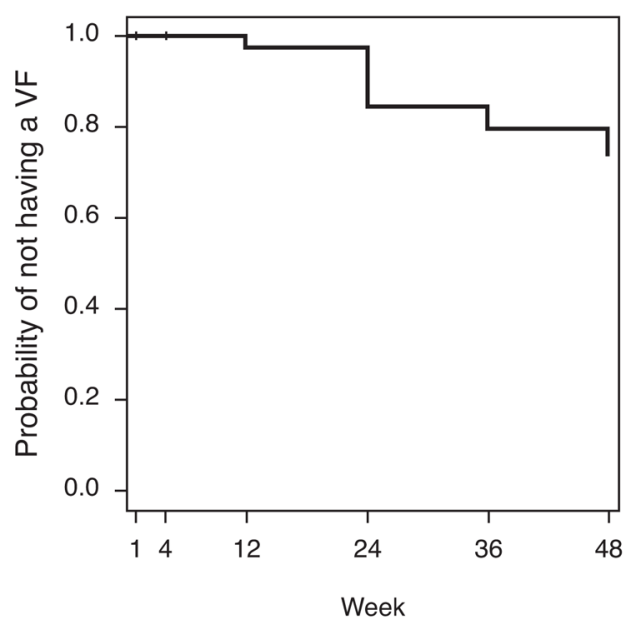

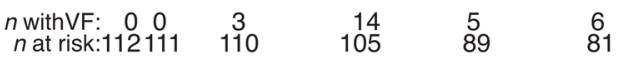

(b) By baseline HIV-1 RNA

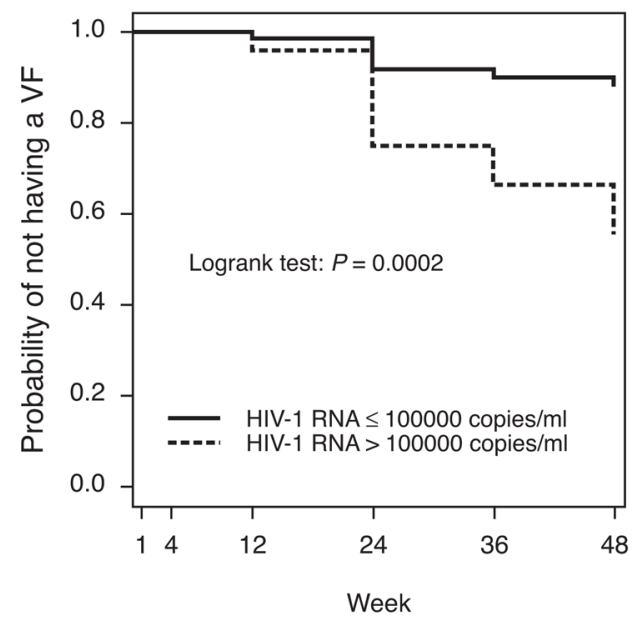

HIV-1 RNA $\leq 100000$ copies/ml

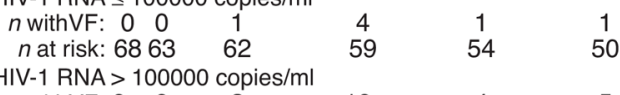

HIV-1 1 RN

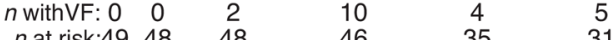

Fig. 2.

Kaplan-Meier plots of time to virologic failure (VF) using intent-to-treat approach. 


\section{Table 1}

Baseline characteristics of participants who initiated darunavir/r plus raltegravir $(n=112)$ by virologic failure status.

\begin{tabular}{|c|c|c|c|c|}
\hline \multirow[b]{2}{*}{ Characteristic } & \multirow[b]{2}{*}{ Total } & \multicolumn{2}{|c|}{ VF status } & \multirow[b]{2}{*}{$P$} \\
\hline & & With VF & Without VF & \\
\hline \multicolumn{5}{|l|}{ Age (in years) } \\
\hline$N$ & 112 & 28 & 84 & $0.835^{*}$ \\
\hline Mean (SD) & $37(11)$ & $37(13)$ & $37(11)$ & \\
\hline Median (Q1-Q3) & $36(27-45)$ & $35(26-44)$ & $36(28-45)$ & \\
\hline $18-29$ & $33(29 \%)$ & $9(32 \%)$ & $24(29 \%)$ & $0.760^{* * *}$ \\
\hline $30-39$ & $38(34 \%)$ & $7(25 \%)$ & $31(37 \%)$ & \\
\hline $40-49$ & $25(22 \%)$ & $7(25 \%)$ & $18(21 \%)$ & \\
\hline $50-59$ & $12(11 \%)$ & $4(14 \%)$ & $8(10 \%)$ & \\
\hline$\lcm{60}$ & $4(4 \%)$ & $1(4 \%)$ & $3(4 \%)$ & \\
\hline \multicolumn{5}{|l|}{ Sex } \\
\hline Male & $98(88 \%)$ & $25(89 \%)$ & $73(87 \%)$ & $1.000^{* *}$ \\
\hline Female & $14(13 \%)$ & $3(11 \%)$ & $11(13 \%)$ & \\
\hline \multicolumn{5}{|l|}{ Race/ethnicity } \\
\hline White non-Hispanic & $49(44 \%)$ & $10(36 \%)$ & $39(46 \%)$ & $0.618^{* *}$ \\
\hline Black non-Hispanic & $45(40 \%)$ & $14(50 \%)$ & $31(37 \%)$ & \\
\hline Hispanic (regardless of race) & $16(14 \%)$ & $4(14 \%)$ & $12(14 \%)$ & \\
\hline Asian, Pacific Islander & $2(2 \%)$ & $0(0 \%)$ & $2(2 \%)$ & \\
\hline \multicolumn{5}{|l|}{ IV drug history } \\
\hline Never & $102(91 \%)$ & $26(93 \%)$ & $76(90 \%)$ & $0.194^{* *}$ \\
\hline Currently & $1(1 \%)$ & $1(4 \%)$ & $0(0 \%)$ & \\
\hline Previously & $9(8 \%)$ & $1(4 \%)$ & $8(10 \%)$ & \\
\hline \multicolumn{5}{|l|}{ Baseline CD4 (cells/ $\mu 1)$} \\
\hline Mean (SD) & $284(199)$ & $199(167)$ & $312(202)$ & $0.007^{*}$ \\
\hline Median (Q1-Q3) & $271(107-419)$ & $192(51-310)$ & $322(147-442)$ & \\
\hline$<200$ & $40(36 \%)$ & $15(54 \%)$ & $25(30 \%)$ & $0.018^{* * * * *}$ \\
\hline 200 to $<350$ & $32(29 \%)$ & $8(29 \%)$ & $24(29 \%)$ & \\
\hline 350 to $<500$ & $26(23 \%)$ & $3(11 \%)$ & $23(27 \%)$ & \\
\hline 2500 & $14(13 \%)$ & $2(7 \%)$ & $12(14 \%)$ & \\
\hline \multicolumn{5}{|c|}{ Baseline HIV-1 RNA ( $\log _{10}$ copies/ml) } \\
\hline Mean (SD) & $4.83(0.60)$ & $5.14(0.61)$ & $4.73(0.57)$ & $0.002^{*}$ \\
\hline Median (Q1-Q3) & $4.87(4.33-5.31)$ & $5.22(4.85-5.60)$ & $4.70(4.31-5.16)$ & \\
\hline \multicolumn{5}{|l|}{ Baseline HIV-1 RNA (copies/ml) } \\
\hline 5000 & $3(3 \%)$ & $1(4 \%)$ & $2(2 \%)$ & $0.006^{* * *}$ \\
\hline 5001 to $\leq 10000$ & $5(4 \%)$ & $1(4 \%)$ & $4(5 \%)$ & \\
\hline 10001 to $\leq 100000$ & $55(49 \%)$ & $5(18 \%)$ & $50(60 \%)$ & \\
\hline 100001 to 5750000 & $43(38 \%)$ & $18(64 \%)$ & $25(30 \%)$ & \\
\hline
\end{tabular}




\begin{tabular}{ccccc}
\hline & & \multicolumn{2}{c}{ VF status } & \\
\cline { 3 - 4 } Characteristic & Total & With VF & Without VF & $P$ \\
\hline$>750000$ & $6(5 \%)$ & $3(11 \%)$ & $3(4 \%)$ & \\
\hline
\end{tabular}

IV, intravenous; VF, virologic failure.

* Exact Wilcoxon test.

** Fisher's exact test.

**** Cochran-Armitage Test. 


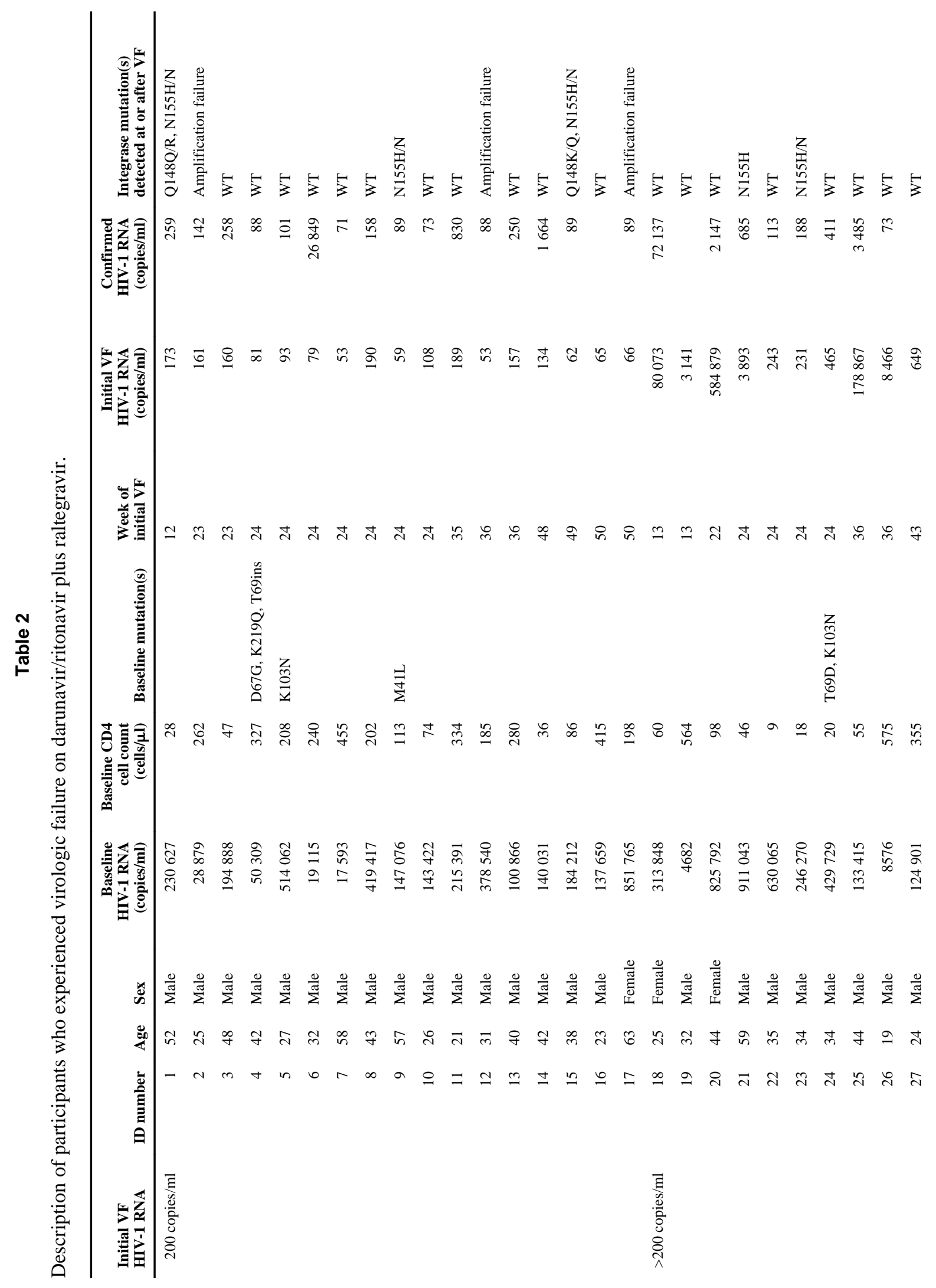

AIDS. Author manuscript; available in PMC 2012 December 05. 


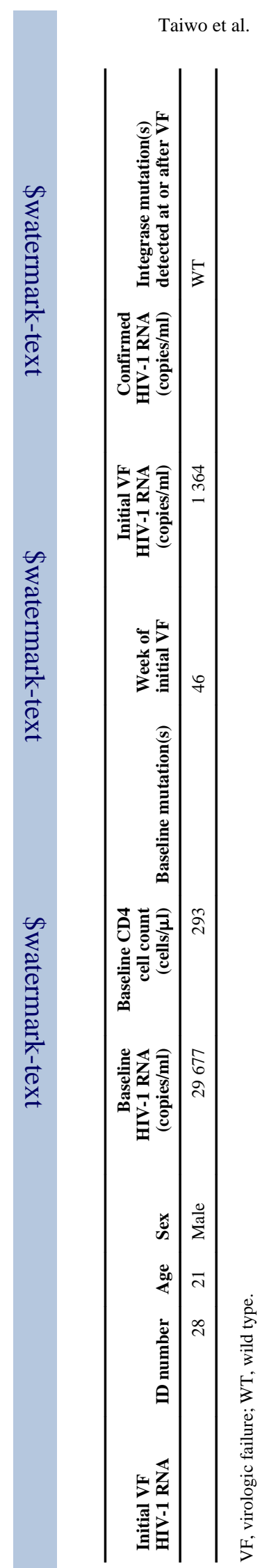

Page 17 AIDS. Author manuscript; available in PMC 2012 December 05. 
Table 3

Trough concentration (categorical) by virologic failure status. ${ }^{a}$

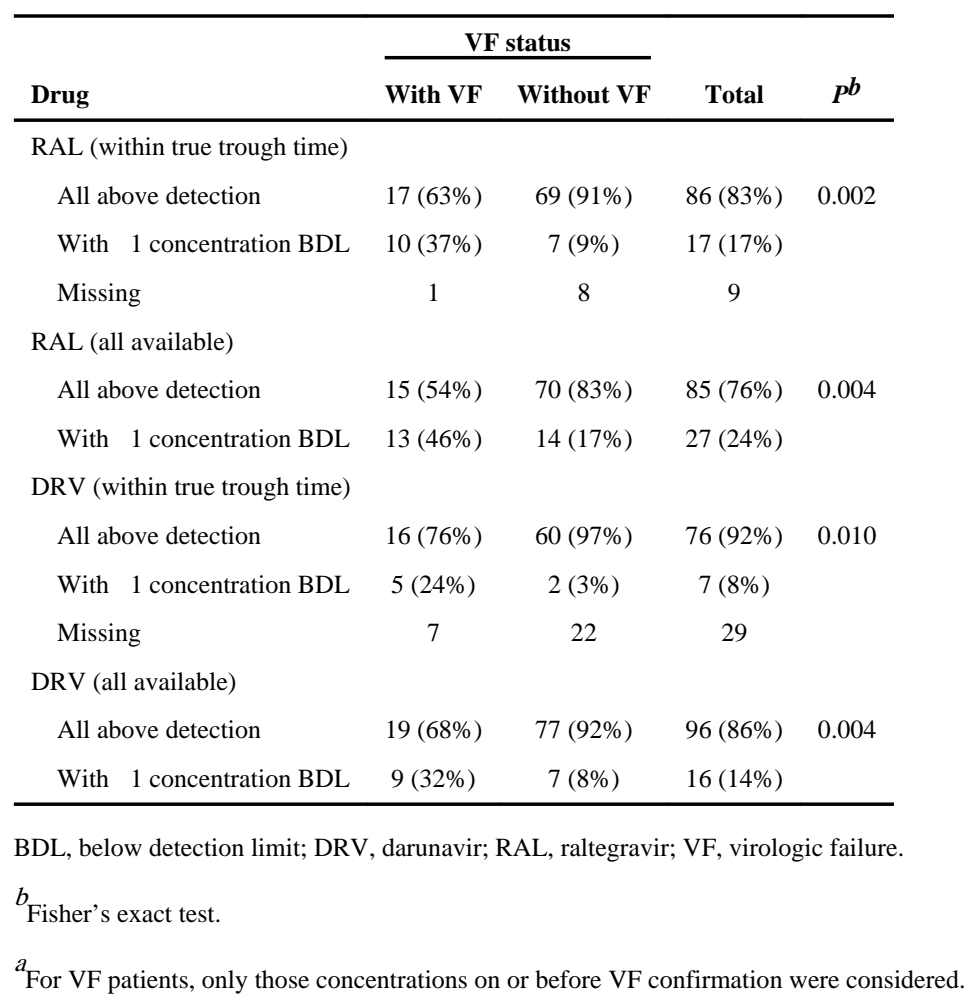

